Raus aus dem Hamsterrad

\title{
Zeitpolitik als feministisches Politikfeld
}

\author{
Die Ressource Zeit ist ungleich zwischen \\ den Geschlechtern verteilt. Dies kann als eine \\ der zentralen Ungerechtigkeiten innerhalb \\ der Gesellschaft aufgefasst werden. Welche Rolle \\ können Geschlechteraspekte in der Zeitpolitik \\ spielen? \\ Von Gesine Agena
}

D ie Frage, wie in unserer Gesellschaft Zeit verteilt ist, beschäftigt schon seit langer Zeit Feministinnen, Öko-Aktivist/innen, Wachstumskritiker/innen, Wissenschaftler/innen und viele andere. Sie diskutieren über die Aufteilung von Zeit und über die Möglichkeit einer neuen Verteilung dieser Ressource. Aber was genau meint eigentlich Zeitpolitik als Politikfeld? Meines Erachtens geht es in erster Linie um eine neue Verteilung von Zeit, Macht und Geld zwischen den Geschlechtern. Denn hier besteht eine der zentralen Ungerechtigkeiten innerhalb der Gesellschaft. Aber es geht auch um eine Gerechtigkeitsfrage insgesamt. Und gerade im politischen Kontext kommt man um eine andere Arbeitsmarkt-, Familien- und Pflegepolitik nicht herum.

Eine zentrale Frage bei der Zeitverteilung ist die Geschlechter(un)gerechtigkeit. Lebenszeit, Arbeitszeit - zu Hause und am Arbeitsplatz -, Zeit mit Kindern, im Haushalt, mit Freund/ innen und Verwandten: Diese Zeiten sind zwischen Männern und Frauen ungleich verteilt. Unser Wirtschafts- und Gesellschaftsmodell beruht darauf, dass Männer tendenziell Vollzeit und Frauen oft nur Teilzeit oder gar nicht erwerbsarbeiten (siehe dazu den Beitrag von Sabine Reiner in diesem Heft). Dafür übernehmen sie den Großteil der unbezahlten Sorge- und Pflegearbeit zu Hause. Dieses Modell wird milliardenschwer vom Staat gefördert. Das Ehegattensplitting beispielsweise fördert mit 20 Milliarden Euro im Jahr diese Eineinhalb-Ernährer-Familie.

\section{Geschlechtergerechtigkeit als zentrale Frage}

Was gegen dieses Modell spricht, ist erstens die Tatsache, dass weder Mütter noch Väter heute so leben wollen. $76 \%$ der Eltern finden, beide Elternteile sollten gleichermaßen verantwortlich für die Erziehung ihrer Kinder sein. Immerhin $63 \%$ der Männer sagen, dass das auch gegeben sei. Im krassen Gegensatz dazu sehen das nur 36\% der Frauen genauso (Menkens 2015). Es gibt in Deutschland also den großen Wunsch von Müttern wie Vätern, Kindererziehung gleichberechtigt untereinander aufzuteilen. Die Möglichkeit dazu hat aber kaum jemand.

Was zweitens gegen das aktuelle System spricht, sind die Konsequenzen, die daraus erwachsen. Im Vergleich zu ihren Vollzeit erwerbstätigen, meistens männlichen Kollegen, haben Frauen oft geringere Chancen auf eine Karriere. Zusätzlich sind es meist Frauen, die wegen ihres geringen Arbeitsvolumens keine eigene Absicherung durch fehlende oder geringe Rentenansprüche haben. Mag sich das alte System, inklusive Ehegattensplitting, im Hier und Jetzt noch lohnen, so zeigen sich später oft schlimme Konsequenzen, die Frauen nicht selten in Altersarmut bringen. Und auch hier wird sichtbar, dass viele Menschen sich schon heute etwas anderes wünschen: Der Anteil von Müttern, die in Vollzeit arbeiten, bleibt gering, etwa bei $40 \%$, auch wenn $75 \%$ der Mütter angeben, dass es ihnen wichtig oder sogar sehr wichtig sei, berufstätig zu sein (Reimann 2015). Gründe genug, das aktuelle System zu ändern!

Aus geschlechterpolitischer Sicht ist Zeit also ungleich verteilt. Männer haben im Schnitt deutlich mehr Zeit für Erwerbsarbeit, Frauen deutlich mehr Zeit für unbezahlte Care-Arbeit.

Zeitpolitik muss Frauen daher eine eigenständige Existenzsicherung ermöglichen, damit Altersarmut verhindert werden kann und Frauen ein selbstbestimmtes Leben führen können. Dazu gehören zum Beispiel die Abschaffung des Ehegattensplittings, ausreichend Betreuungsplätze und ein Ende der Minijobs, die keine Absicherung bieten. Grüne Zeitpolitik will Familien, Müttern, Vätern und Alleinerziehenden ermöglichen, so zu leben, wie sie es sich wünschen und vielfach nicht können.

Auch jenseits der Geschlechterungleichheit ist Zeit in unserer Gesellschaft ungerecht verteilt. Während ein Teil der Bevölkerung immer mehr arbeitet, ist ein anderer Teil fast völlig ausgeschlossen von guter Arbeit. Viele haben mehr als einen Job, um mit dem Einkommen gerade so über die Runden zu kommen.

\section{Zeitpolitik umsetzen}

Eine gute Zeitpolitik muss daher auch diese Ungerechtigkeiten beseitigen. Das bedeutet, dass politische Maßnahmen sozial ausgewogen sein müssen. Einige Menschen in den oberen Einkommensbereichen können sich eine Reduzierung ih- 
rer Arbeitszeit schon heute leisten. Andere können über so etwas nicht einmal nachdenken, weil ihre Existenz daran hängt, dass sie ihre zwei oder drei Jobs behalten. Eine gerechte Zeitpolitik kommt daher um Umverteilung nicht herum.

Aber wie werden aus diesen Grundsätzen und aus der Unzufriedenheit über das bestehende System politische Handlungsoptionen? Innerhalb der Grünen Partei und unserer Bundestagsfraktion arbeitet seit knapp zwei Jahren eine Gruppe aus Fachpolitiker/innen an Ideen, wie wir eine neue Zeitpolitik umsetzen können. Einige der Ideen skizziere ich hier.

\section{Ein neues Bild von Vollzeit}

Warum bildet die 40-Stunden-Woche eigentlich unseren Standard? Jeden Tag acht Stunden arbeiten, wenn es gerade brennt, Überstunden machen - da bleibt nicht viel Zeit für Familie, Freund/innen, ehrenamtliches Engagement oder zum Ausruhen und Abschalten. Durch die Digitalisierung sind wir immer und überall erreichbar. Viele checken und bearbeiten auch nach Feierabend ihre Arbeits-Mails. Personen, die viel arbeiten wollen und können, sollen dies tun. Menschen, für die eine Vollzeitstelle zum Genickbruch wird, müssen aber bessere Möglichkeiten haben, beruflich kürzerzutreten.

Dafür müssen wir die Rechte von Arbeitnehmer/innen stärken. Mit unseren Instrumenten einer grünen Zeitpolitik wollen wir einen rechtlichen Rahmen für eine flexiblere Mitbestimmung über die eigene Arbeitszeit schaffen. Das heißt beispielsweise, dass wir eine andere Präsenzkultur für Beschäftigte etablieren wollen, damit sie ihre Arbeitszeit flexibler an den eigenen Bedürfnissen und Verpflichtungen orientiert einteilen können. Ein Instrument dazu können Arbeitszeitkorridore sein, in welchen die Erwerbsarbeitszeit zwischen 30 und 40 Stunden variieren kann. Innerhalb von Arbeitszeitkorridoren sollen Beschäftigte ihren Arbeitszeitumfang bedarfsgerecht bestimmen können. Damit wird die Grenze zwischen Teilzeit und Vollzeit durchlässiger. Zudem setzen wir uns neben dem Recht auf Teilzeit für ein Rückkehrrecht auf Vollzeit ein, um es insbesondere für Eltern leichter möglich zu machen, in ihre vorherige Anstellung zurückzukehren, wenn ihre Kinder älter sind. Insgesamt zielt die grüne Zeitpolitik darauf ab, ein neues Bild von Vollzeit zu etablieren.

\section{Zeit für Familie}

Ein wichtiges Ziel unserer Zeitpolitik ist es, Familien zu unterstützen. Denn unter anderem zeigt eine 2015 durch das Forsa-Institut durchgeführte Studie der Zeitschrift „Eltern“, dass mehr als $60 \%$ aller Eltern sich unter Druck fühlen und auch viele Kinder wahrnehmen, dass ihre Eltern gestresst sind.

Darum arbeiten wir an dem Modell einer neuen Familienzeit, zu der wir das Elterngeld verlängern wollen. Ein Elternteil soll jeweils die Möglichkeit haben, zwölf Monate vollständig aus dem Beruf auszusteigen oder alternativ anteilig Elterngeld zu beziehen, wenn der Wiedereinstieg in den Job mit einer reduzierten Stundenzahl geschafft ist. Damit wollen wir Eltern mehr Zeit für die Familie und gleichzeitig Frauen einen schnelleren Wiedereinstieg in den Job ermöglichen.

Aber Menschen brauchen nicht nur Zeit, wenn es um die Erziehung von Kindern geht. Auch wenn die Eltern pflegebedürftig werden, sind Auszeiten notwendig, um sich um alles kümmern zu können. Daher arbeiten wir auch an einem Modell für eine Pflegezeit. Damit wollen wir eine bis zu dreimonatige Freistellung für Menschen ermöglichen, die sich um die Versorgung pflegebedürftiger Angehöriger oder Bekannter kümmern. So wird ihnen mit einer Lohnersatzleistung die Möglichkeit gegeben, sich um die Organisation der Pflege zu kümmern, den Pflegebedarf einzuschätzen, sich über Leistungsangebote und -ansprüche $\mathrm{zu}$ informieren, diese zu beantragen und die jeweils notwendigen Hilfen zu organisieren.

\section{Politikfeld mit viel Potenzial}

Zeitpolitik ist ein Thema, in dem viel Bewegung und Potenzial zur Veränderung steckt. Es ist ein feministisches Politikfeld, da es zentrale Ungleichheiten zwischen den Geschlechtern anspricht und die Möglichkeit bietet, daran etwas zu ändern. Die hier genannten Ideen sind nur einige der Modelle, die wir Grüne aktuell entwickeln, um aus dem Thema der Zeitpolitik politische Handlungsoptionen abzuleiten.

Eine neue Zeitpolitik kann und sollte aber nicht nur vom Staat verordnet oder festgelegt werden. Es geht ganz grundsätzlich um einen Diskurs, den wir als Gesellschaft führen sollten: Wie gehen wir mit unserer Zeit um? Welches Wirtschaftsmodell wollen wir? Wem steht viel freie Zeit zur Verfügung und wem wenig? Wer kann selbstbestimmt über Zeit verfügen? Wie organisieren wir Zeitwohlstand für alle Menschen? Zeitpolitik bietet die Möglichkeit, diese Fragen zu stellen und für mehr Gerechtigkeit zu streiten.

\section{Literatur \\ Menkens, S. (2015): Mütter und Väter in Deutschland machen sich selbst viel zu viel Druck. In: Die Welt online, 13.01.2015. \\ Reimann, A. (2015): Allensbach-Studie: Deutsche Eltern halten's konservativ. In: Spiegel Online, 07.07. 2015.}

